\title{
Experimental Investigations of Cochannel Interference Reduction Effect at High Elevation Base Station Using Beam Tilt and Orthogonal Polarization
}

\author{
Shuta Uwano, ${ }^{1}$ Atsuya Ando, ${ }^{1}$ Tomohiro Seki, ${ }^{2}$ Yasushi Takatori, ${ }^{2}$ and Takefumi Hiraguri ${ }^{3}$ \\ ${ }^{1}$ Access Network Service Systems Laboratories, NTT Corporation, Yokosuka 239-0847, Japan \\ ${ }^{2}$ Network Innovation Laboratories, NTT Corporation, Yokosuka 239-0847, Japan \\ ${ }^{3}$ Faculty of Electrical and Electronics Engineering, Nippon Institute of Technology, Miyashiro 345-8501, Japan \\ Correspondence should be addressed to Shuta Uwano; ueno.shuta@lab.ntt.co.jp
}

Received 22 July 2013; Revised 18 November 2013; Accepted 2 December 2013; Published 8 January 2014

Academic Editor: Miguel Ferrando

Copyright ( 2014 Shuta Uwano et al. This is an open access article distributed under the Creative Commons Attribution License, which permits unrestricted use, distribution, and reproduction in any medium, provided the original work is properly cited.

\begin{abstract}
This paper addresses the problem of cochannel interference (CCI) generated in a mixed cell architecture in microcellular systems. In this type of microcellular systems in which both microcells and macrocells coexist in the same geographical urban area, the base station antennas mounted on the rooftops of buildings to cover wide circular radio zones suffer severe CCI from the surrounding low base stations. A dielectric-loaded slotted-cylinder antenna (DSCA) is applied to horizontally polarized omnidirectional array antennas in a height-diversity configuration with the high gain of $8 \mathrm{dBi}$, which is comparable to that of a collinear antenna, to reduce the CCI. The measurements conducted in a suburban area clarify the reduction in the CCI for three techniques. The beamtilt technique reduces the CCI level by approximately $10 \mathrm{~dB}$ for both collinear antennas and the DSCA array antennas. The use of horizontal polarization reduces the CCI level by approximately $13 \mathrm{~dB}$ for the DSCA array antennas with and without beam tilt. The combination of the beam tilt and horizontal polarization or the DSCA array antennas with beam tilt significantly reduces the CCI level by approximately $23 \mathrm{~dB}$.
\end{abstract}

\section{Introduction}

The number of subscribers to the personal handy-phone system (PHS) [1], which was developed and successfully implemented in Japan as a wireless personal communication service (PCS), has continued to increase beyond 80 million mostly in middle South American, African, and Asian countries especially China [2]. PHS supplies not only public wireless services but also wireless local loop (WLL) and fixed wireless access (FWA) systems [3] and has recently been applied to new services such as logistics [4] and ETC [5].

In a PHS system with a street microcell [6], all base station (BS) antennas are installed at locations lower than the surrounding buildings. The buildings form a radio zone called a street microcell along the street. As a fundamental concept in PHS design, the street microcell does not assume the occurrence of cochannel interference (CCI) between BSs. This is because the buildings act as obstacles and as a result increase the propagation loss compared to that of elevated BSs because of the low BS height. Attention should be paid to the fact that commercial PHS with a street microcell basically uses vertically polarized antennas; therefore, the polarization of the CCI waves is mainly vertical as reported in [7]. One of the disadvantages of the PHS street microcell is that numerous BSs are needed to cover all service areas and installing many BSs is costly. When we construct commercial systems, we frequently face a rather exceptional situation in conjunction with the expansion of PHS service areas. PHS adopts a cell architecture called a mixed cell architecture [8] in which both microcells and macrocells coexist in the same geographical area using high-elevation BS antennas to form circular macrocells, even though constructing high elevation $\mathrm{BSs}$ is contrary to the original PHS design principle.

In a PHS system with a macrocell, BS antennas with an omnidirectional radiation pattern and high gain such as collinear antennas are usually mounted at a high location 


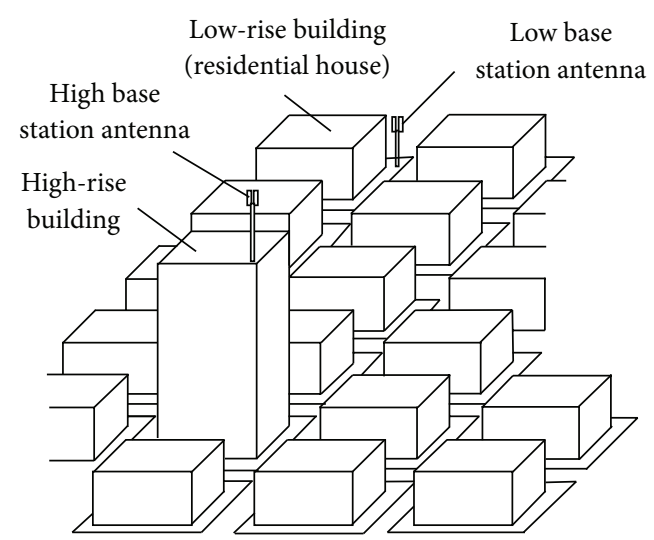

FIGURE 1: Cochannel interference between high base station and low base station antennas in TDMA/TDD system.

such as the rooftop of a building to cover a wide area with circular radio zones as shown in Figure 1. Such an elevated BS antenna experiences severe CCI from other surrounding BS antennas. This is because the propagation loss of the CCI decreases and no obstacles exist at the level of the BS receiving the interference (hereafter interference receiving base station (IRBS)) to block the CCI when the antennas of the IRBS are mounted at high locations such as a rooftop. As a consequence of these situations, an IRBS in a PHS system set on a rooftop cannot find an available time slot in the time division multiple access/time-division duplex (TDMA/TDD) scheme by employing autonomous distributed control and they cannot transmit control channel $(\mathrm{CCH})$ signals because all the time slots are frequently occupied by the CCI with higher levels than the carrier sense level of PHS.

It is well known that the easiest and most economical method to suppress CCI is the beam down-tilt technique [913]. This technique, which generates nulls in the horizontal plane, is often used to reduce the CCI and was adopted in many BS antennas in both cellular and microcellular wireless communication systems throughout the world. Another method to reduce CCI for high-elevated BSs in a macrocell is the autonomous decentralized synchronization technique $[14,15]$, which synchronizes time slots in TDMA/TDD between BSs to lower the CCI and increase the channel capacity. Unfortunately, these techniques offer only limited success and a more effective approach is still needed. As an alternative method to suppress the CCI, adaptive array antennas were newly proposed and shown to suppress the CCI for elevated PHS BSs [7]. However, the adaptive array antennas used in this configuration are rather expensive compared to the conventional BS antennas for commercial systems.

The adoption of horizontally polarized diversity antennas with beam tilt is another method to reduce the CCI further. This method provides both polarization orthogonal to the polarization of the CCI waves and beam tilt effects. When horizontally polarized diversity antennas that have a high peak gain comparable to that of collinear antennas are used for such a high elevation BS, the following items should be studied or clarified: the CCI reduction effect and the radio zone length (RZL) with respect to the inclination angle of the mobile antenna based on measurements. These two research topics are of great significance to the design and development of commercial microcell systems. To the best knowledge of the authors, there is no report that shows the CCI reduction effect based on empirical data when the three techniques, that is, beam tilt, use of orthogonal-polarization, and a combined technique of beam tilt/orthogonal-polarization, are employed for BS antennas.

This paper addresses the problem of the CCI generated in a mixed cell architecture in PHS that adopts the TDMA/TDD scheme by using horizontally polarized omnidirectional array antennas in a height-diversity configuration that permits PHS BS antennas established at high elevations to form a macrocell and cover a wide area with circular radio zones. In association with the CCI reduction technique using horizontally polarized diversity antennas for IRBSs, the concept of a polarization arrangement for the BS antennas in a mixed cell architecture is introduced. We developed a dielectric-loaded slotted-cylinder antenna (DSCA) array that has a horizontally polarized omnidirectional radiation pattern with a high gain that is comparable to that of a collinear antenna. We applied it to dual-polarized omnidirectional diversity antennas to compensate for the deterioration in the received signal due to a mismatch in the polarization direction between the base station antenna and mobile station antenna [16]. In this paper, the DSCA array is applied to horizontally polarized omnidirectional array antennas in a height-diversity configuration to reduce the CCI. The measurements conducted in a suburban area clarify the change in the distribution of interfering BSs (IBSs) and the reduction effect in the CCI for three techniques: beam tilt, the use of horizontally polarized antennas, and a combination of the beam tilt and horizontally polarized antennas. The impact of the inclination angle of the mobile antenna on the RZL of the DSCA array is shown based on measurements on an urban street.

Section 2 describes the CCI reduction technique that employs horizontally polarized antennas with beam tilt for the IRBS and a polarization arrangement of BS antennas in a mixed cell architecture. It also describes a method for estimating the RZL with respect to the inclination angle of the mobile antenna. In Section 3, the measurement results of the CCI reduction effect achieved by the DSCA array antenna are shown for the comparison to the CCI level of a collinear antenna. Section 4 shows the RZL variations in the DSCA array and collinear antennas with respect to the inclination angle of the mobile antenna. Section 5 discusses the results from this study and future work. Finally, our conclusions are presented in Section 6.

\section{CCI Reduction Technique, Polarization Arrangement in Mixed Cell Architecture, and RZL Estimation Method}

2.1. CCI Reduction Technique and Polarization Arrangement in Mixed Cell Architecture. The CCI reduction technique using polarization orthogonal to the polarization of the CCI 


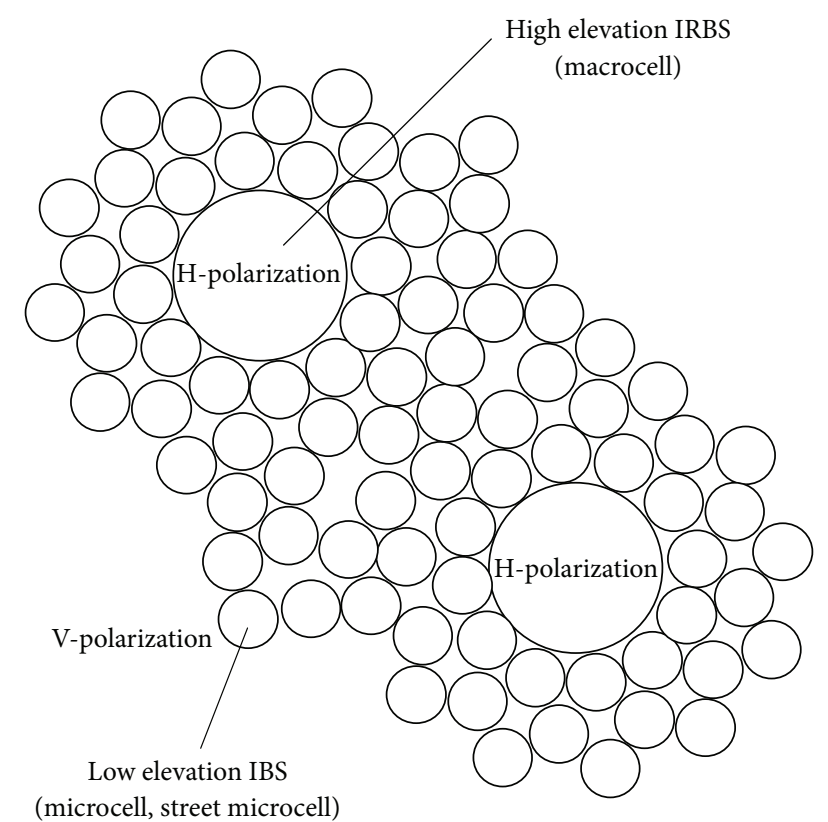

FIGURE 2: High and low elevation base station antenna arrangement sharing vertical and horizontal polarization.

waves and a high and low base station antenna arrangement sharing vertical and horizontal polarization are shown in Figure 2. In the mixed cell architecture considered in this study, which is contrary to the current architecture, an IRBS at a high elevation separately uses horizontally polarized omnidirectional antennas with/without beam tilt to cover a circular radio zone to form macrocells. It is expected that horizontally polarized diversity antennas with beam tilt will most effectively reduce the CCI level due to the combined effect of both the horizontal polarization and beam tilt.

Polarization diversity is the most effective solution to compensate for the deterioration in the received signal due to a mismatch in the polarization direction between the BS antenna and mobile station antenna, and dual-polarized antennas were proposed to minimize the degradation in the received signal strength for both PHS BS antennas in high [16] and low elevation BSs [17]. It should be emphasized that this paper investigates the IRBS set on a rooftop in propagation environments in which the polarization diversity technique cannot be applied because of the following reasons: severe CCI as described in [7]; because the diversity branch of a vertically polarized antenna of a dual-polarized diversity antenna receives high-level vertically polarized CCI waves; because an available time slot cannot be found in TDMA/TDD; and because a link cannot be established between the BS and user terminals no matter how the other branch, a horizontally polarized antenna, lowers the CCI level.

2.2. RZL Estimation Method. It has been generally believed that the RZL should be estimated with respect to only the angle at which polarization matching between the BS and mobile antennas is satisfied. Since mobile handy phones

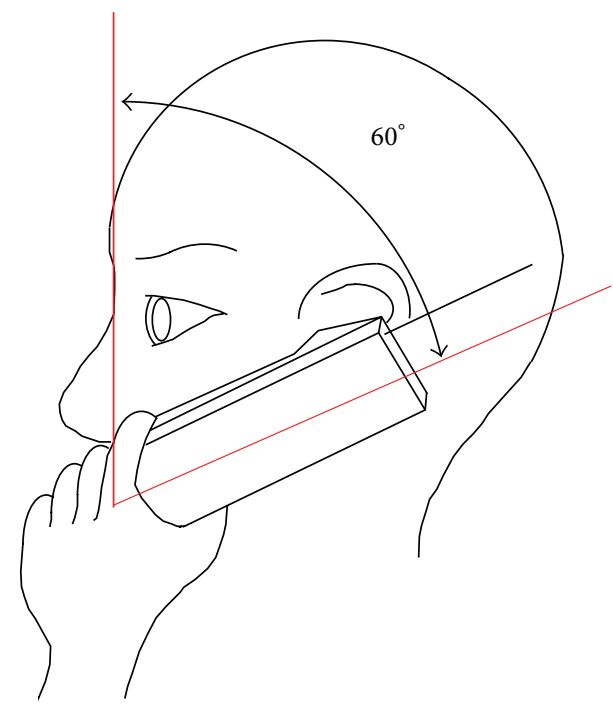

FIGURE 3: Typical inclination angle of handy phone while talking.

equipped with whip antennas or built-in antennas are inclined at various angles when people use them, the received signal level at the BS also degrades due to a mismatch in the polarization direction between the BS antenna and mobile station antenna [18]. It is of more significance to resolve the degradation in the received signal level caused by a mismatch in the polarization direction between the BS antenna and mobile station antenna rather than the degradation in the gain of the radiation patterns caused by user proximity or the absorption of radiation power by the human body. The degradation of the former is greater than that of the latter [19]. It was also reported that the average inclination angle based on a statistical distribution of the handy phones in use is approximately $60^{\circ}$ as shown in Figure 3 [20]. In this paper, we compare RZLs based on two aspects: one is the averaging value when the mobile antennas are slanted from $0^{\circ}$ to $90^{\circ}$, and the other is with respect to the specific value when the mobile antennas are slanted at $60^{\circ}$ as described in [20].

\section{Interference Reduction Effect}

3.1. Measurement Scenario. The measuring point is on the rooftop of a $16 \mathrm{~m}$ high building in the vicinity of a railway station in suburban commercial and residential areas. There are no obstacles around the building at the IRBS antenna level. The view from the rooftop is shown in Figure 4. The IBS antennas are collinear antennas with no beam tilt, have the peak gain of $8 \mathrm{dBi}$ and the transmit power of $20 \mathrm{~mW}$, and transmit a vertically polarized wave. It should be noted that all CCI waves are vertically polarized. The collinear antennas are set on power or telegraph poles at the height of approximately $7 \mathrm{~m}$ to $8 \mathrm{~m}$ above ground level. We chose the measurement site where the CCI is the most severe, that is, where the antenna height is higher than the rooftop levels of two-story residential houses. Therefore, the condition of the antenna height is inconsistent with the street-microcell design; however, the measured results are considered to be the 


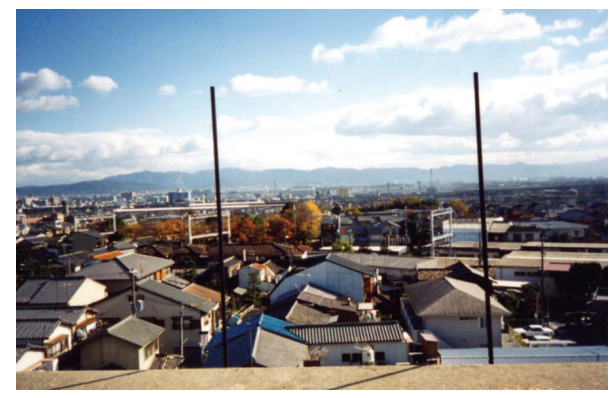

Figure 4: View toward Fushimi district shown in Figures 10 and 11 from the rooftop at which cochannel interference was measured.

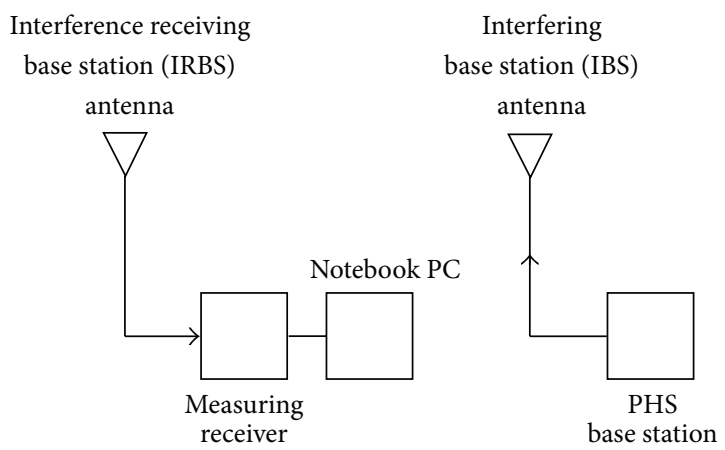

FIGURE 5: Block diagram of CCI measurement equipment.

worst estimation of the CCI. The IBSs are distributed widely in locations that straddle suburban areas in the cities of Kyoto, Mukou, and NagaokaKyo in Japan as shown in the maps (Figures 10 and 11). The CCI levels were measured for the top branch of the diversity branches and diversity reception was not applied. The control channel (CCH) signal at $1.916 \mathrm{GHz}$ is used for CCI level measurements. A measurement receiver (PHS Measuring receiver ML5661A, Anritsu Corporation, Japan) was used to receive the $\mathrm{CCH}$ signals. A block diagram of the CCI measurement equipment is shown in Figure 5. In this study, the effects of interference reduction were statistically estimated using cumulative distribution curves of the received CCI levels for each IRBS antenna configuration. We did not analyze the details of each propagation path between individual IBS antennas and the IRBS antenna on the rooftop. We also did not confirm line-of-sight (LOS) propagation conditions between each IBS antenna and the IRBS antenna.

We developed a DSCA array antenna that has a horizontally polarized omnidirectional radiation pattern with a high gain that is comparable to that of a collinear antenna and applied it to dual-polarized omnidirectional diversity antennas [16]. The peak gain is approximately $8 \mathrm{dBi}$ and is comparable to that of the conventional 4-element collinear antennas. The down-tilt angle is approximately $15^{\circ}$. It should be noted that these array antennas are designed for the commercial $1.9 \mathrm{GHz}$ band in this measurement. The specifications of the DSCA prototype and conventional collinear antenna are summarized in Table 1 . In this study,
TABLE 1: Specifications of DSCA prototype and conventional collinear antenna.

\begin{tabular}{lcc}
\hline & DSCA & Collinear \\
\hline Diameter of radome & $22 \mathrm{~mm}$ & $17 \mathrm{~mm}$ \\
Number of elements & 4 & 4 \\
Gain & $8 \mathrm{dBi}$ & $8 \mathrm{dBi}$ \\
Polarization & Horizontal & Vertical \\
Down-tilt angle & $15^{\circ} / 0^{\circ}$ & $15^{\circ} / 0^{\circ}$ \\
Radiation pattern & Omni & Omni \\
\hline
\end{tabular}

we applied the DSCA array to horizontally polarized omnidirectional antennas with a high gain at elevated PHS BSs mounted on rooftops of buildings to form a circular radio zone. Conventional collinear antennas without beam tilt (V_antennas), conventional collinear antennas with beam tilt (V_tilt_antennas), DSCA array antennas without beam tilt (H_antennas), and DSCA array antennas with beam tilt (H_tilt_antennas) were used to measure the CCI levels. The radiation patterns for V_antennas, V_tilt_antennas, H_antennas, and H_tilt_antennas are shown in Figures 6, 7, 8 , and 9 , respectively.

3.2. Distribution of Interfering BSs. The changes in distribution of the IBS antennas are mapped in Figures 10 and 11 . We should note that the total number of IBSs is 48 and signals from all the BSs are certainly received by at least one of four antenna configurations. Since the minimum received signal sensitivity level of the measurement receiver is approximately $20 \mathrm{~dB} \mu \mathrm{V}$, the IBSs for which the CCI levels are less than $20 \mathrm{~dB} \mu \mathrm{V}$ are not shown in the figures. Figures 10 and 11 show that the distribution areas and the number of IBS antennas become progressively smaller corresponding to the order of V_antenna, V_tilt_antenna, H_antenna, and H_tilt_antenna. The CCIs from the IBS antennas installed in street microcells in the urban area around the JR Kyoto Station are not received at all due to blocking by a group of high-rise buildings, while the CCI from the IBS established on the famous Kiyomizu Temple in the mountainous Higashiyama district as indicated by the arrow in Figure 10 is received at an exceptionally high level because the measuring point and the temple are unintentionally in LOS propagation. The relationship between the received CCI levels versus the distances from the IRBS to the IBSs is shown in Figure 12. The changes in the numbers of visible IBSs, the IBSs among the visible IBSs at which the CCI levels received by the IRBSs are higher than the carrier sense level of $26.5 \mathrm{~dB} \mu \mathrm{V}$, and the farthest distance of the IBS from the measuring point for the four types of antenna configurations are summarized in Table 2. The distribution areas, the number of IBSs, and the received CCI level become progressively smaller corresponding to the order of V_antenna, V_tilt_antenna, H_antenna, and H_tilt_antenna. Considering the farthest distance of $9400 \mathrm{~m}$ from the IBS at the temple as an exception, the farthest distance of $1500 \mathrm{~m}$ from the IRBS to an IBS when using the H_tilt_antenna is approximately less than $23 \%$ of the farthest distances from the IRBS to IBS of when 


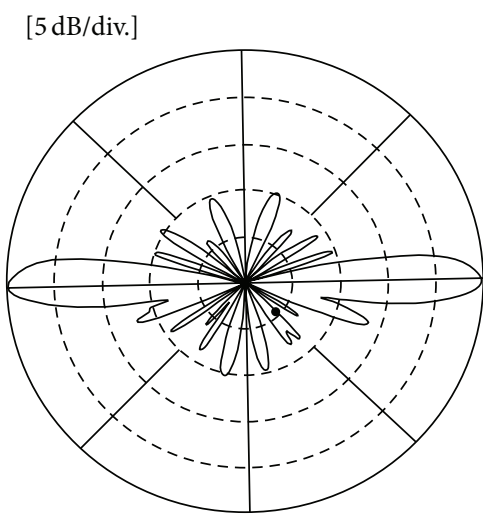

(a)

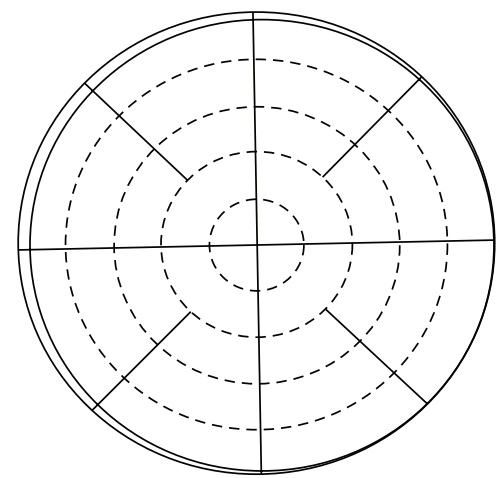

(b)

FIGURE 6: Measured radiation patterns of collinear antennas (V_antennas). (a) Vertical plane. (b) Horizontal plane.

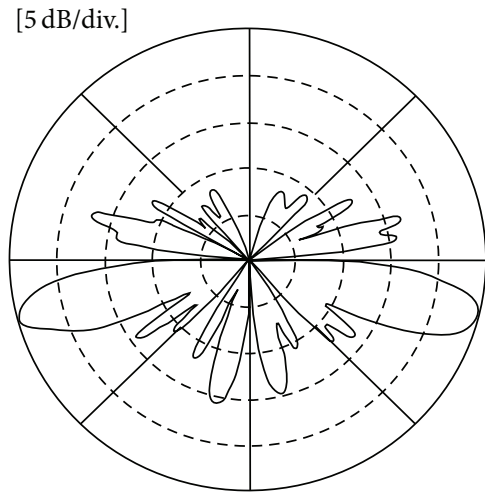

(a)

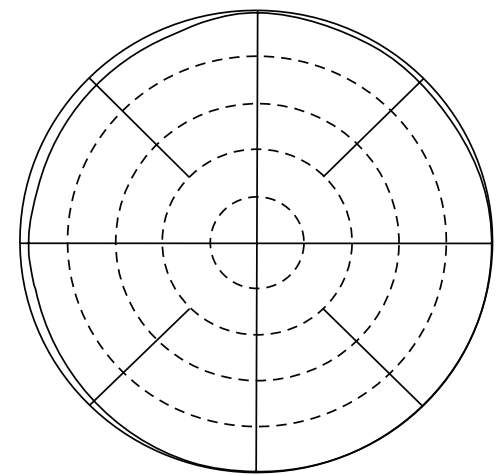

(b)

FIGURE 7: Measured radiation patterns of collinear antenna with beam tilt (V_tilt_antennas). (a) Vertical plane. (b) Conical plane.

using the V_tilt_antenna and V_antenna. It is clear that the H_tilt_antenna effectively decreases the CCI compared to the H_antenna and V_tilt_antenna. However, it is interesting to compare the difference in the CCI reduction effect between the V_tilt_antenna and $\mathrm{H}_{-}$antenna. The numbers of visible IBSs and IBSs at which the received CCI level is greater than the carrier sense level are decreased from $32(66.7 \%)$ and 27 (56.3\%) to $23(47.9 \%)$ and 17 (35.4\%) for the V_tilt_antenna and H_antenna, respectively. The farthest distances from the measuring point to an IBS are decreased from $6500 \mathrm{~m}$ to $2116 \mathrm{~m}$ for the V_tilt_antenna and $\mathrm{H}_{-}$antenna, respectively. These results show that the H_antenna is superior to the V_tilt_antenna in decreasing the CCI.

3.3. Interference Reduction Effect. The cumulative distribution curves of the received CCI levels for the four types of antenna configurations are shown in Figure 13. It should be noted that the invisible IBSs in Figures 10 and 11, defined for those with received $\mathrm{CCH}$ levels of less than $20 \mathrm{~dB} \mu \mathrm{V}$ (the minimum received sensitivity level of the measurement receivers), are newly included as samples to each cumulative distribution. Therefore, the total number of samples in each cumulative distribution should be 48 as described
TABLE 2: Numbers of IBSs and the farthest distances to IBSs.

\begin{tabular}{lccc}
\hline $\begin{array}{l}\text { Antenna } \\
\text { configuration }\end{array}$ & $\begin{array}{c}\text { Number of } \\
\text { visible IBSs }{ }^{* 1}\end{array}$ & $\begin{array}{c}\text { Number of } \\
\text { IBSs where received } \\
\text { CCI level is greater } \\
\text { than carrier sense } \\
\text { level }^{* 2}(\%)\end{array}$ & $\begin{array}{c}\text { Farthest } \\
\text { distances to } \\
\text { IBSs (m) }\end{array}$ \\
\hline V & $38(79.2 \%)$ & $38(79.2 \%)$ & $6500(9400)$ \\
V_tilt & $32(66.7 \%)$ & $27(56.3 \%)$ & 6500 \\
H & $23(47.9 \%)$ & $17(35.4 \%)$ & 2116 \\
H_tilt & $11(22.9 \%)$ & $6(12.5 \%)$ & 1500 \\
${ }^{* 1}$ Total number of IBSs established in locations that straddle suburban areas \\
$\begin{array}{l}\text { is 48. } \\
{ }^{* 2} \text { Carrier sense level is } 26.5 \mathrm{~dB} \mu \mathrm{V} .\end{array}$
\end{tabular}

in Section 3.2. All the received CCI levels of the invisible IRBSs are assumed to be constant at $20 \mathrm{~dB} \mu \mathrm{V}$ assuming the worst case of CCI. The CCI reduction effects are estimated at $80 \%$ probability of the cumulative distribution curves of the CCI because the measured data are valid and used for the estimation. The beam-tilt technique reduces the CCI level by approximately $10 \mathrm{~dB}$ for both the V_tilt_antenna and H_tilt_antenna. The use of horizontal polarization reduces the 


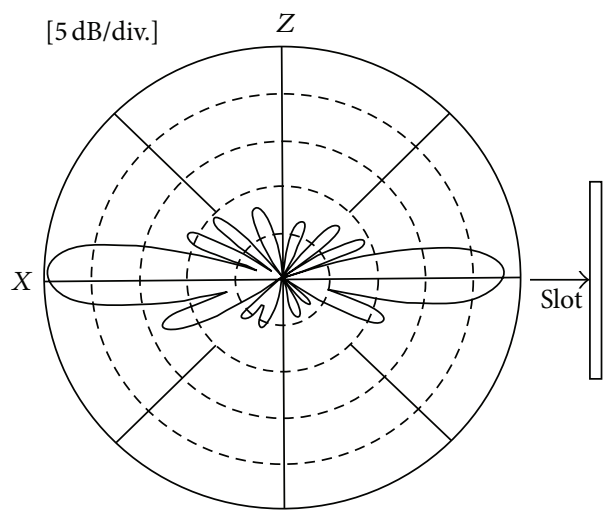

(a)

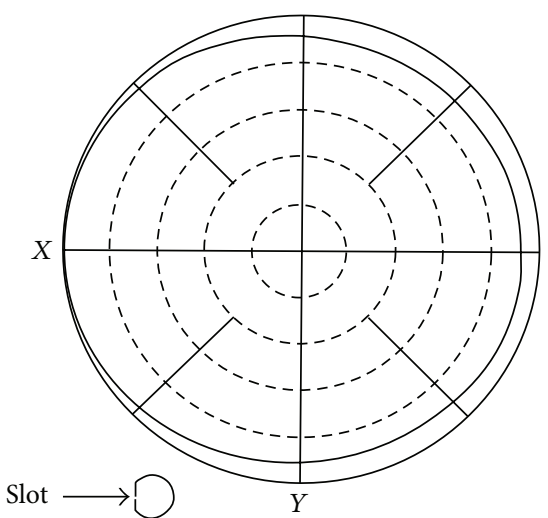

(b)

Figure 8: Measured radiation patterns of the four-element DSCA array (H_antennas). (a) Vertical plane. (b) Horizontal plane.

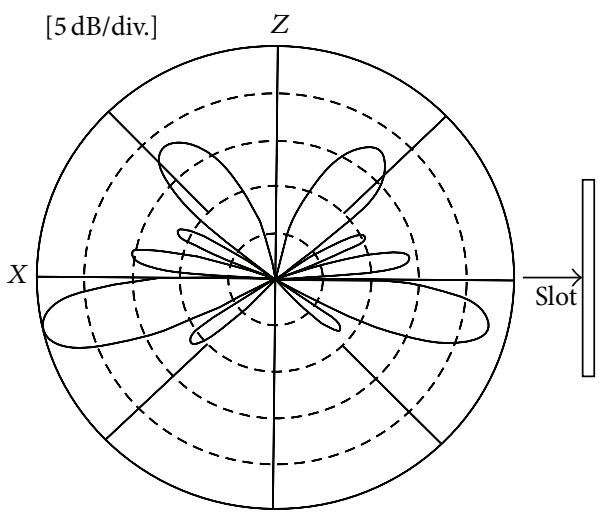

(a)

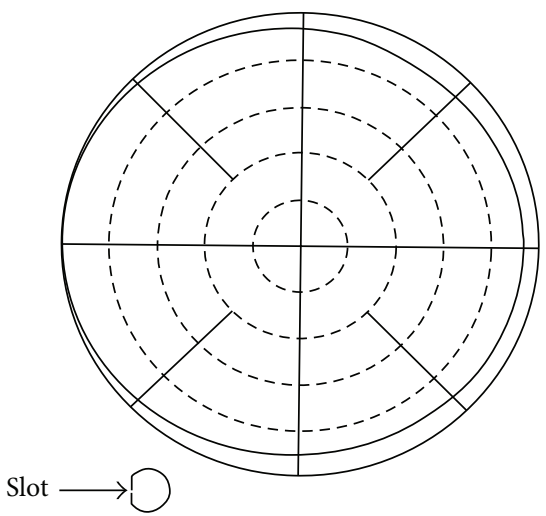

(b)

FIGURE 9: Measured radiation patterns of the four-element DSCA array with beam tilt (H_tilt_antennas). (a) Vertical plane. (b) Conical plane.

CCI level by approximately $13 \mathrm{~dB}$ for both the H_tilt_antenna and H_antenna. We confirm that the polarization effect is superior to the beam-tilt effect in reducing the CCI, and when only the beam-tilt technique is employed the CCI is insufficiently suppressed. The combined technique of the beam tilt and horizontal polarization reduces the CCI level by approximately $23 \mathrm{~dB}$ for the H_tilt_antenna. The CCI reduction effect when employing the H_antenna is only approximately $3 \mathrm{~dB}$ greater than the CCI reduction effect for the V_tilt_antenna, while the farthest distance from the measuring point to the interfering BS when using the H_antenna is approximately $23 \%$ of that when using the V_tilt_antenna as explained in Section 3.2. We confirm again that the H_antenna is superior to the V_tilt_antenna with respect to reducing the CCI. We note that the measurements on the CCI reduction effect were conducted in suburban or residential areas; however, we also confirmed the effect in dense urban areas in Osaka City by adopting the technique to BS antennas in commercial operation.

\section{Radio Zone Length Measurement}

4.1. Measurement Scenario. The measurements of the RZLs for the four types of height-diversity antenna configurations were conducted on Yonegahama street in an urban area in Yokosuka city. Figure 14 shows a scenario for measuring the RZLs on this urban street and the definition of the inclination angle in LOS environments. Figure 15 shows the view from the location of the base station antenna. A map of the area surrounding the BS antennas and measurement routes in LOS environments are shown in Figure 16. The transmitting mobile antenna, a sleeve antenna at the height of $1.6 \mathrm{~m}$, with the transmitting power of $25.85 \mathrm{dBm}$ was mounted on a cart. The cart moved along the sidewalk of the $25 \mathrm{~m}$ wide street on the opposite side from a $30 \mathrm{~m}$ high building in the LOS environments to determine the RZL. The receiving $\mathrm{BS}$ antennas were set on the rooftop of the building, and measurement receivers (Measuring Receiver ML524B, Frequency Converter MH669B, Anritsu Corporation, Japan) were used to receive the signals. The received signal levels were processed using selection diversity and the regression fitting curves were calculated. A block diagram of the RZL measurement equipment is shown in Figure 17. The length of the LOS route was approximately $630 \mathrm{~m}$. The measured frequency was $2.2 \mathrm{GHz}$. The proposed horizontally polarized diversity antennas were compared to regular collinear diversity antennas. The sleeve antenna representing the mobile antenna was inclined in the $Z-X$ plane in LOS as depicted 


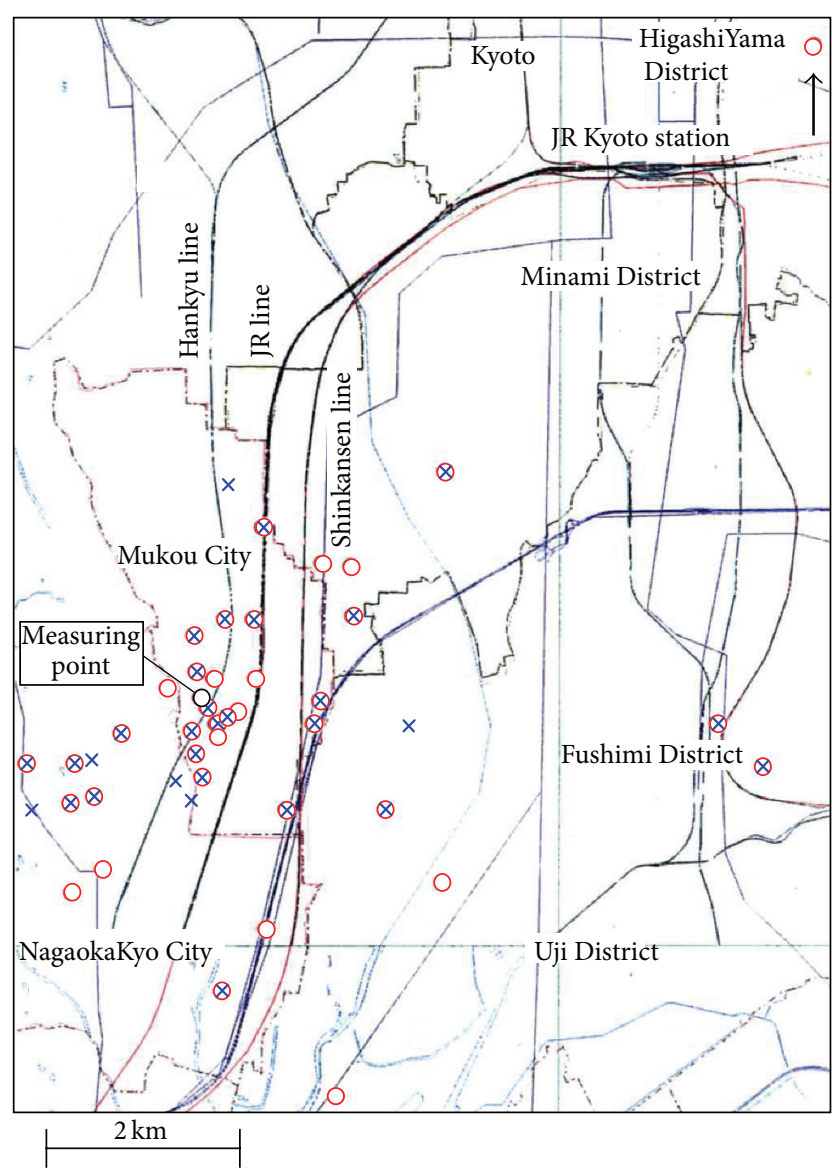

$\circ \mathrm{V}$

$\times$ V_tilt

FIGURE 10: Distribution of IBSs when IRBS antennas are V_antennas and V_tilt_antennas.

TABLE 3: RZLR for four types of diversity antennas.

\begin{tabular}{lcc}
\hline $\begin{array}{l}\text { Diversity antenna } \\
\text { configuration }\end{array}$ & Average RZLR (\%) & RZLR at $60^{\circ}(\%)$ \\
\hline V & 60.5 & 47.6 \\
V_tilt & 40.2 & 36.7 \\
H & 66.6 & 78.3 \\
H_tilt & 62.8 & 70.7 \\
\hline
\end{tabular}

in Figure 14(b). Approximately 6300 median values of the received signal were considered. One median value for every $0.1 \mathrm{~m}$ is calculated so that the original received signal over a $1 \mathrm{~m}( \pm 0.5 \mathrm{~m})$ interval was sampled at $100 \mathrm{kHz}$.

4.2. Radio Zone Length. The received signal levels for V_antenna/H_antenna and V_tilt_antenna/H_tilt_antenna are shown in Figures 18 and 19, respectively. The received signal levels for a collinear antenna with no beam tilt for $0^{\circ}$ and $90^{\circ}$ are shown in Figure 18 and are the same as those in Figure 12 in [16]. In PHS, the acceptable propagation loss is designed to be $99 \mathrm{~dB}$ not accounting for antenna gains for personal and

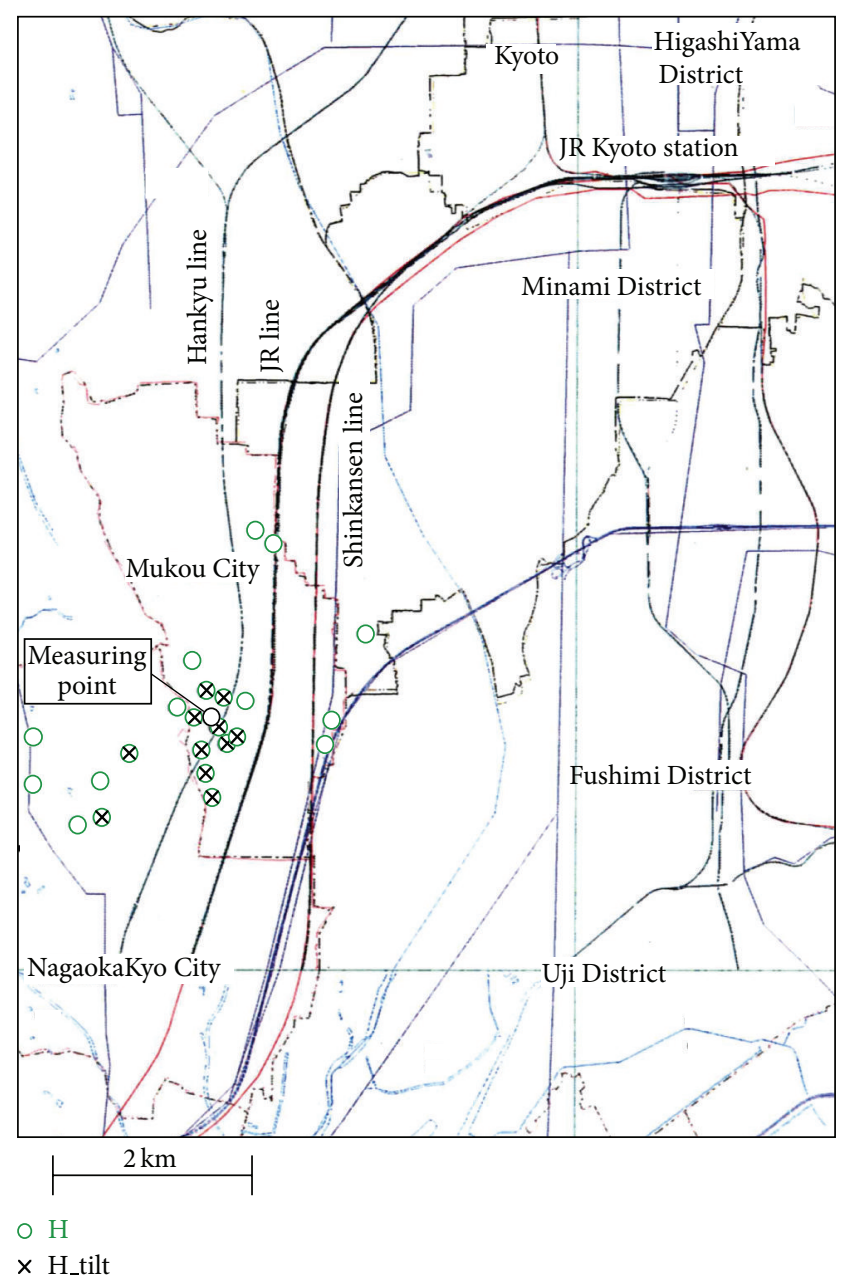

FIGURE 11: Distribution of IBSs when IRBS antennas are H_antennas and H_tilt_antennas.

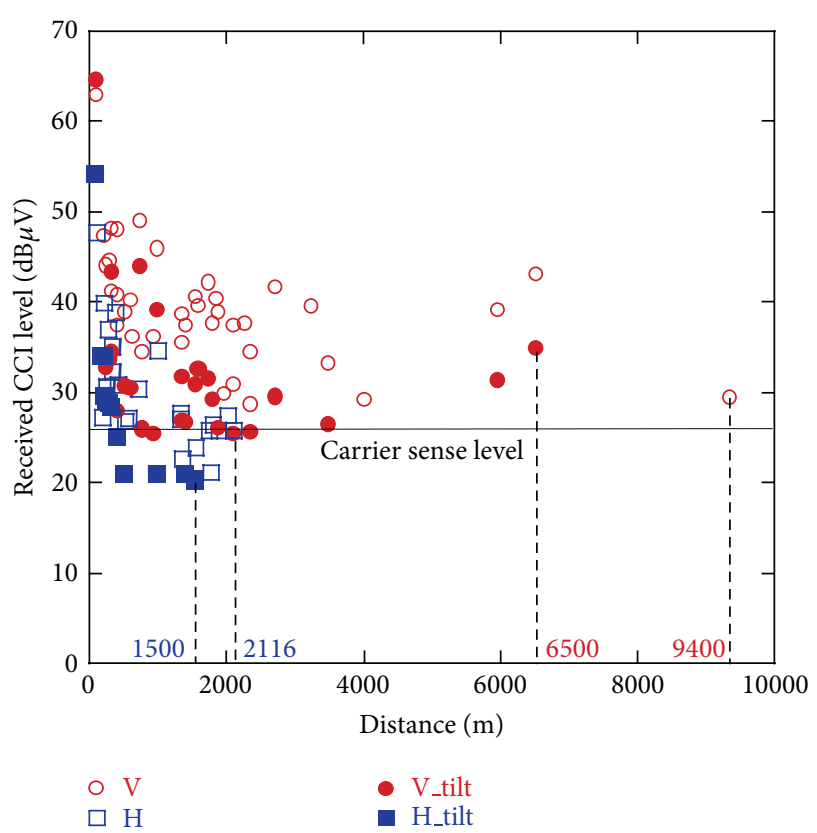

FIGURE 12: Received CCI level versus distance of IBSs. 


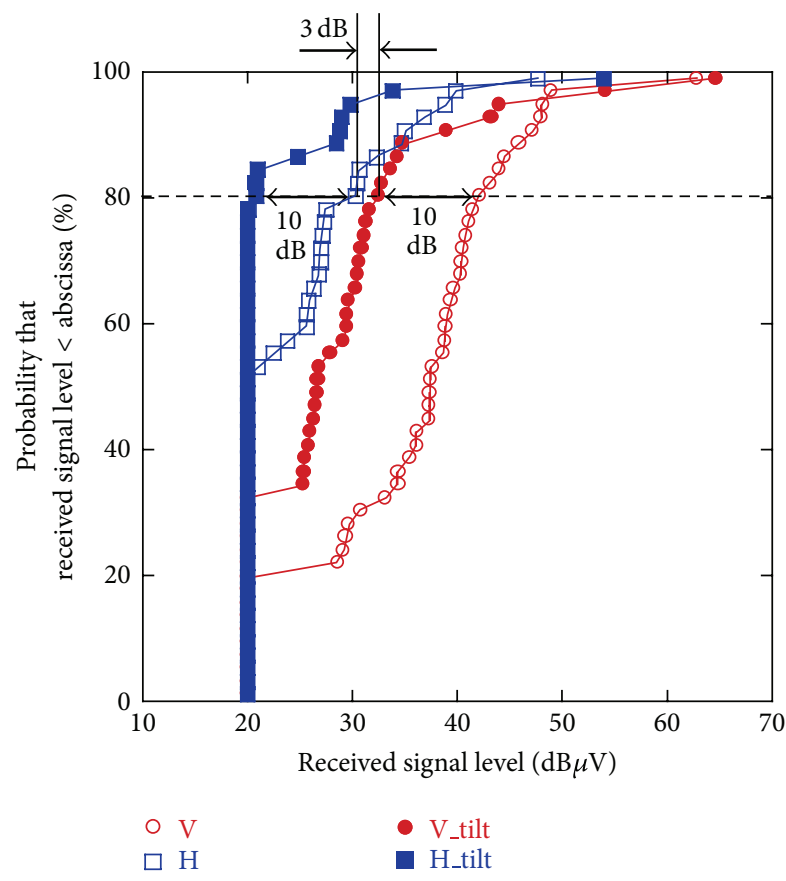

FIGURE 13: Cumulative distribution of the received CCI level.

BS antennas. The transmitting power for this measurement is $25.85 \mathrm{dBm}(138.85 \mathrm{~dB} \mu \mathrm{V})$ and results in $39.85 \mathrm{~dB} \mu \mathrm{V}$ as the threshold. The RZLs are defined such that the regression fitting lines of the received signal level of the BS antennas exceed the threshold level of the system, $39.85 \mathrm{~dB} \mu \mathrm{V}$, when the mobile antenna moved away from the BS antenna. Note that the RZLs are normalized by the RZL of the V_antenna (vertical mobile antenna), that is, $939 \mathrm{~m}$, and are expressed as the RZL ration (RZLR) in percent. The RZLRs of both the V_tilt_antenna/V_antenna and H_tilt_antenna/H_antenna versus the inclination angle of the mobile antenna are shown in Figure 20. The RZLRs for the four types of diversity antennas are summarized in Table 3. The parameters of the regression fitting lines for Figures 18 and 19 are summarized in Table 4. The equation for the regression fitting lines of the received signal levels is of the form:

$$
\mathrm{RSL}=A-B \cdot \log (d),
$$

where RSL is the received signal level in $\mathrm{dB} \mu \mathrm{V}, A$ is the constant, $B$ is the attenuation coefficient, and $d$ is the distance in meters. The threshold level at the end of the radio zone of the system is $39.85 \mathrm{~dB} \mu \mathrm{V}$. The purpose of this study is not to find the break point and a regression fitting line beyond the break point. The distance to the break point is given by an approximate equation using a two-ray model as follows:

$$
R b=\frac{4 h t h r}{\lambda} .
$$

In this RZL measurement, $h t=30 \mathrm{~m}, h r=1.6 \mathrm{~m}, \lambda=$ $0.136 \mathrm{~m}$, and $f=2.2 \mathrm{GHz}$. Therefore, $R b=1412 \mathrm{~m}$ and the RZL values are before the break point.

At approximately $12 \%(111 \mathrm{~m})$ of the RZLR, we observed that the received signal levels of both the V_tiltantenna
TABLE 4: Parameters of regression fitting lines.

(a) V_antenna

\begin{tabular}{lccccc}
\hline \multirow{2}{*}{ Angle $\left(^{\circ}\right)$} & \multirow{2}{*}{$A$} & \multirow{4}{*}{$B$} & \multicolumn{2}{c}{ Range of regression fitting } & \multirow{2}{*}{ RZL (m) } \\
& & & $d_{1}(\mathrm{~m})$ & $d_{2}(\mathrm{~m})$ & \\
\hline 0 & 112.55 & 24.456 & 300 & 630 & 939 \\
45 & 86.69 & 16.708 & 300 & 630 & 636 \\
60 & 108.52 & 25.908 & 300 & 630 & 447 \\
90 & 98.945 & 24.622 & 400 & 630 & 251 \\
\hline
\end{tabular}

(b) V_tilt_antenna

\begin{tabular}{lccccc}
\hline \multirow{2}{*}{ Angle $\left(^{\circ}\right)$} & \multirow{2}{*}{$A$} & \multirow{4}{c}{ Range of regression fitting } & \multirow{2}{*}{ RZL $(\mathrm{m})$} \\
& & & $d_{1}(\mathrm{~m})$ & $d_{2}(\mathrm{~m})$ & \\
\hline 0 & 152.81 & 41.521 & 200 & 630 & 525 \\
45 & 152.62 & 43.412 & 200 & 630 & 396 \\
60 & 153.38 & 44.744 & 200 & 630 & 345 \\
90 & 147.71 & 45.221 & 200 & 630 & 243 \\
\hline
\end{tabular}

(c) H_antenna

\begin{tabular}{lccccc}
\hline \multirow{2}{*}{ Angle $\left(^{\circ}\right)$} & \multirow{2}{*}{$A$} & \multirow{2}{*}{$B$} & \multicolumn{2}{c}{ Range of regression fitting } & \multirow{2}{*}{ RZL (m) } \\
& & & $d_{1}(\mathrm{~m})$ & $d_{2}(\mathrm{~m})$ & \\
\hline 0 & 197.58 & 59.61 & 400 & 630 & 443 \\
45 & 124.33 & 30.13 & 400 & 630 & 637 \\
60 & 120.69 & 28.20 & 400 & 630 & 736 \\
90 & 144.35 & 36.83 & 400 & 630 & 688 \\
\hline
\end{tabular}

(d) H_tilt_antenna

\begin{tabular}{lccccc}
\hline \multirow{2}{*}{ Angle $\left(^{\circ}\right)$} & \multirow{2}{*}{$A$} & \multirow{4}{*}{ Range of regression fitting } & \multirow{2}{*}{ RZL (m) } \\
& & & $d_{1}(\mathrm{~m})$ & $d_{2}(\mathrm{~m})$ & \\
\hline 0 & 111.12 & 27.74 & 200 & 630 & 371 \\
45 & 135.04 & 34.22 & 200 & 630 & 605 \\
60 & 138.66 & 35.01 & 200 & 630 & 664 \\
90 & 125.22 & 29.88 & 200 & 630 & 720 \\
\hline
\end{tabular}

and H_tilt_antenna shown in Figure 19 are increased compared to those of both antennas without beam tilt shown in Figure 18. The RZLR corresponds to the maximum distance at which the beams intersect the road in the measurement routes. Figure 20 shows that the RZLRs of the V_tilt_antenna/V_antenna are decreased by approximately 30 and $73 \%$, respectively, when the mobile antennas are inclined from $0^{\circ}$ to $90^{\circ}$, while the RZLRs of the H_tilt_antenna/H_antenna are increased by approximately 37 and $26 \%$, respectively, when the mobile antennas are inclined from $0^{\circ}$ to $90^{\circ}$. The average RZLRs for the $\mathrm{H}_{-}$antenna/H_tilt antenna are 66.6 and $62.8 \%$ and approximately 1.1- and 1.6fold greater than those for the V_antenna/V_tilt antenna, respectively. It was reported that the average inclination angle distribution of a handy phone in use is approximately $60^{\circ}$ [20]. The RZLRs at a $60^{\circ}$ inclination of the mobile antennas for the H_antenna/H_tilt antenna are 78.3 and $70.7 \%$ and approximately 1.6- and 1.9-fold greater than those for the V_antenna/V_tilt antenna, respectively. The variations in the RZL have not been taken into account in the system design up to now. We conclude from Figure 20 that the $\mathrm{H} \_$antenna/H_tilt antenna is superior to the $\mathrm{V} \_$antenna/V_tilt 


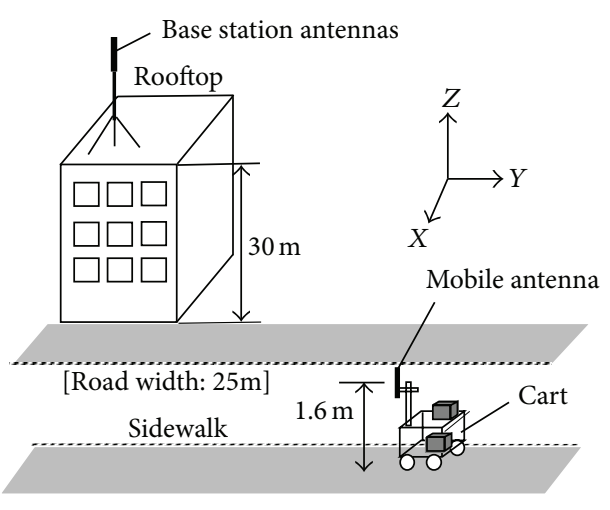

(a)

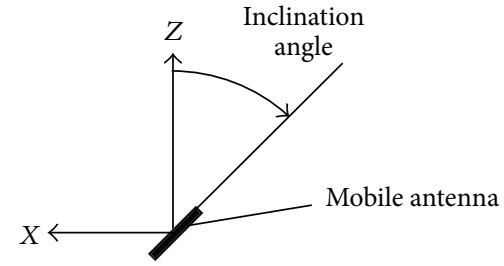

(b)

Figure 14: Scenario of RZL measurement in an urban street. (a) Measurement scenario. (b) Definition of inclination angle.

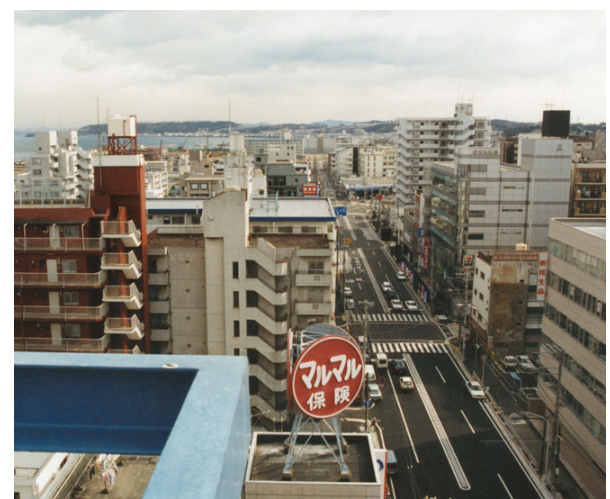

FIGURE 15: View from the rooftop where the RZL was measured.

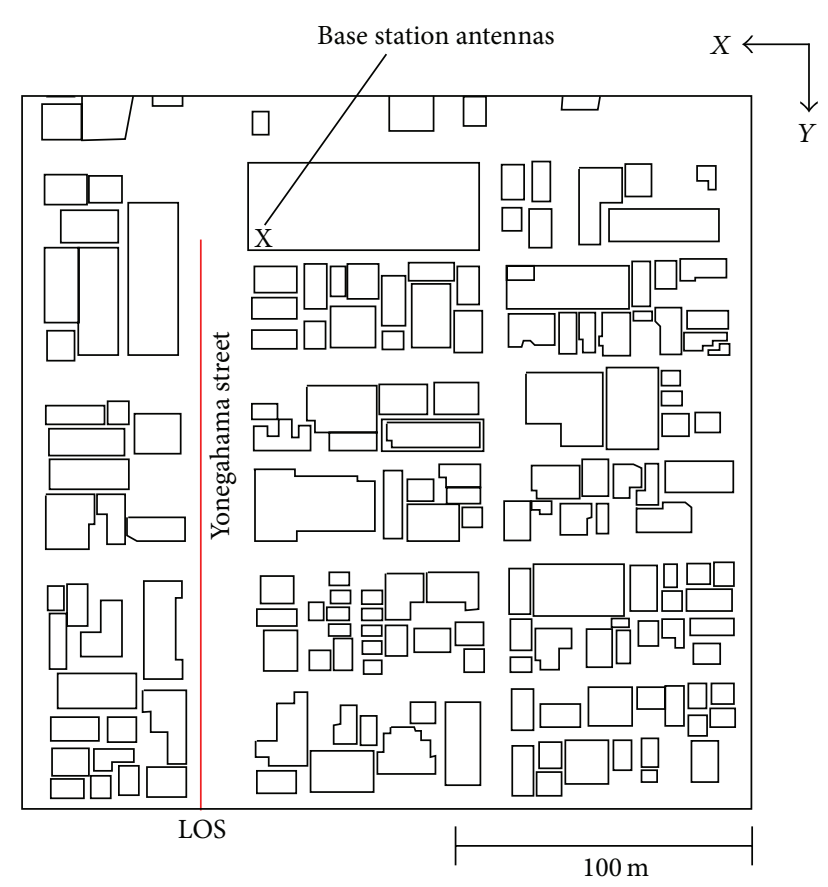

FIGURE 16: Map of measurement route. antenna if we take into account that users do not hold the handset in a strictly vertical position but declined at various angles.

The RZL measurements were conducted in LOS environments in this study. It should be noted that the RZLs in nonline-of-sight (NLOS) environments are short compared to those in LOS environments in microcell systems with a high-elevation BS both when collinear diversity and polarization-diversity antennas are applied to the BS [16].

\section{Discussion}

The two measurements of the CCI reduction level in Section 3 and the RZLs in Section 4 show that the DSCA diversity antennas with a beam tilt reduce the CCI level and simultaneously achieve greater RZLs compared to the RZLs for collinear antennas. The measurements also demonstrate the concepts behind the CCI reduction technique for IRBSs set at high elevations using horizontally polarized diversity antennas with beam tilt and a polarization arrangement of BS antennas in a mixed cell architecture function in an orderly fashion in an actual PHS scenario. We developed DSCA array antennas and applied them to the two types of diversity antennas in our paper: dual-polarized omnidirectional diversity antennas [16] and the horizontally polarized omnidirectional diversity antennas. The reason for using two different types of diversity antennas is that the selection of the diversity antenna configuration depends on the CCI level at the site where the BSs are installed or supposed to be installed. The dualpolarized omnidirectional diversity antennas are preferable for the IRBSs where the CCI levels are so low that the IRBS can find an available time slot in the TDMA/TDD scheme and establish a link between the BS and user terminals, while the horizontally polarized omnidirectional diversity antennas must be applied to the IRBSs where the CCI levels are too high to find an available time slot in the TDMA/TDD scheme and fail to establish a link between the BS and user terminals.

PHS is not the only wireless mobile system employing the TDD scheme. WiMAX, TD-LTE, TD-SCDMA, and iBurst employ this technology. TDD systems will continue to play a key role and be applied to various wireless systems both 


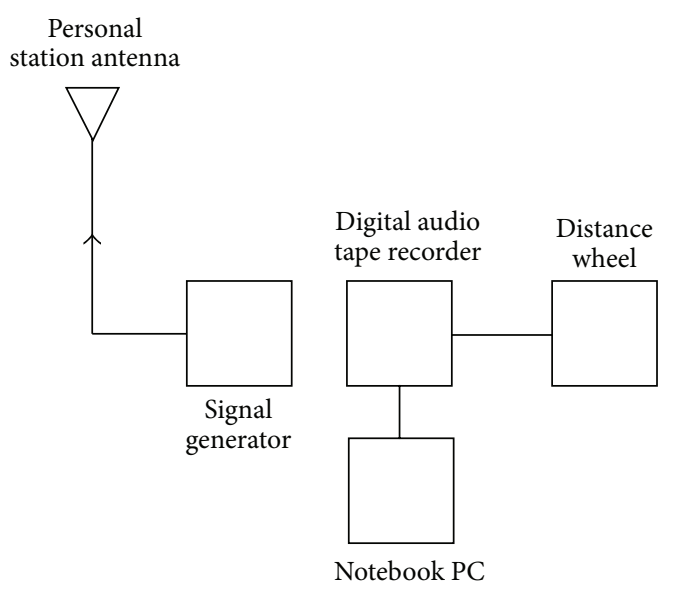

(a)

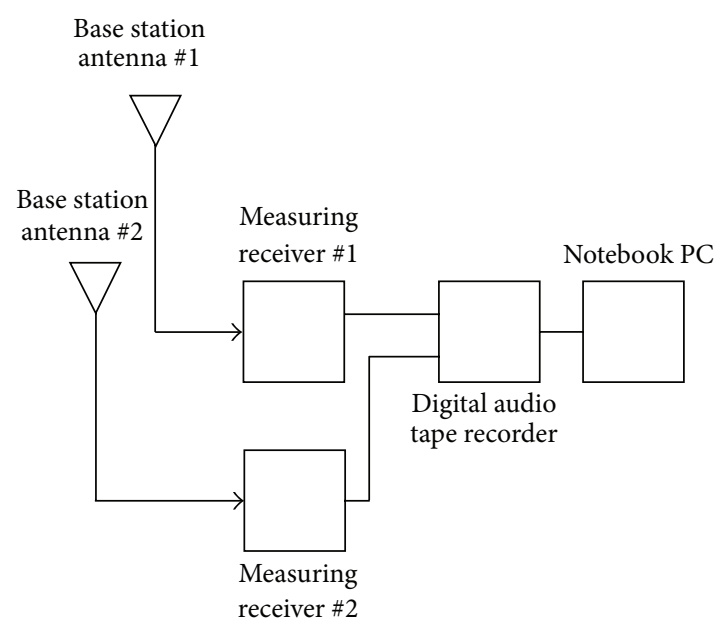

(b)

FIgURE 17: Block diagram of RZL measurement equipment. (a) Personal station. (b) Base station.

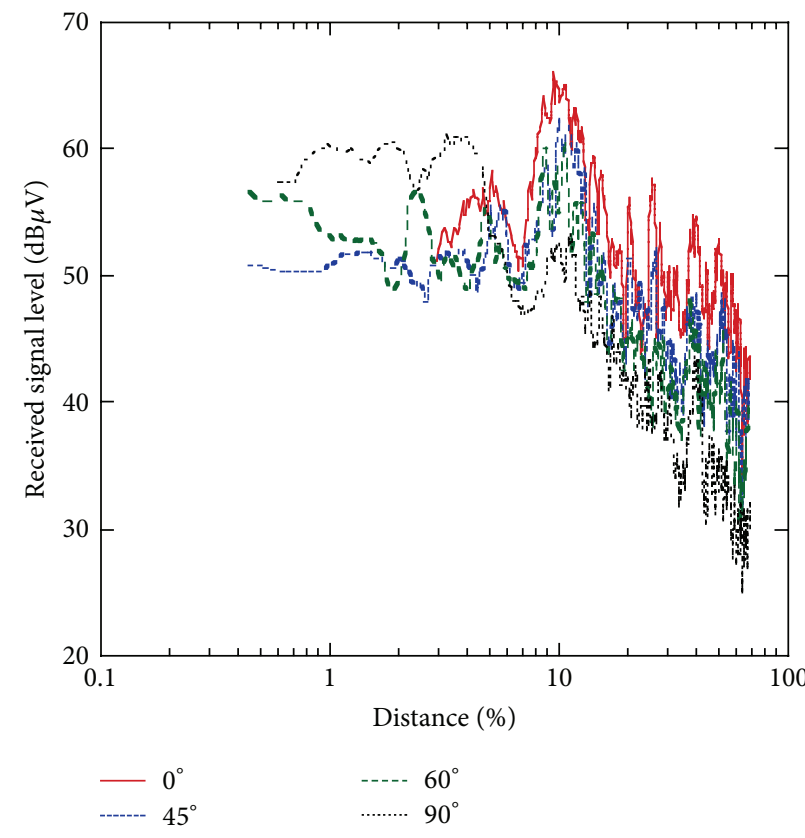

(a)

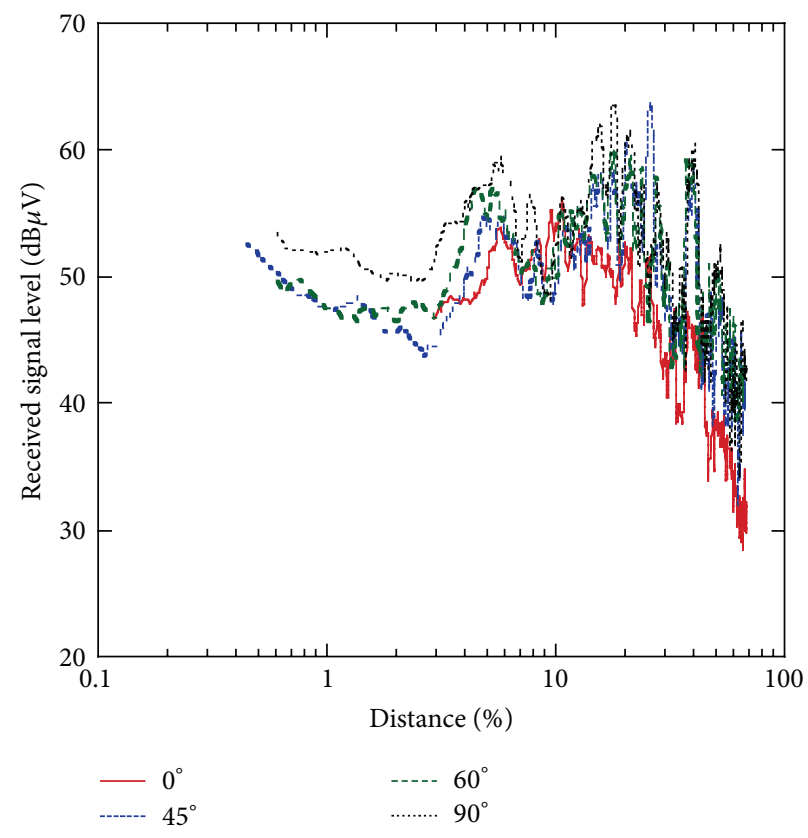

(b)

FIGURE 18: Received-signal level. (a) Collinear with no beam tilt (V_antenna). (b) DSCA array with no beam tilt (H_antenna).

now and in the future. Also, the applicability of the CCI reduction technique does not depend on the transmission scheme such as TDD or FDD. Although we investigated the technique only in a mixed-cell PHS environment, clearly this technique using beam tilt and orthogonal polarization is also applicable to systems comprising conventional microcells or macrocells without mixed-cell structures and reduces the CCI level between adjacent cells.

It has not been made clear which radio wave polarization vertical or horizontal exhibits better characteristics in land mobile communication environments. There have been few studies on the issue. The path gain versus distance characteristics for both vertically and horizontally polarized waves is analyzed based on a two-ray model [21]. This analysis does not take into account the reflected waves from building walls and therefore is insufficient grounds to explain the advantage of the polarization in multipath environments such as street microcells. In this paper, we describe the RZL characteristics for both vertically and horizontally polarized omnidirectional diversity BS antennas when the vertically polarized omnidirectional mobile antenna was inclined. However, these results do not exactly compare the advantage in polarization between vertically and horizontally polarized waves, because the mobile antenna is a vertically 


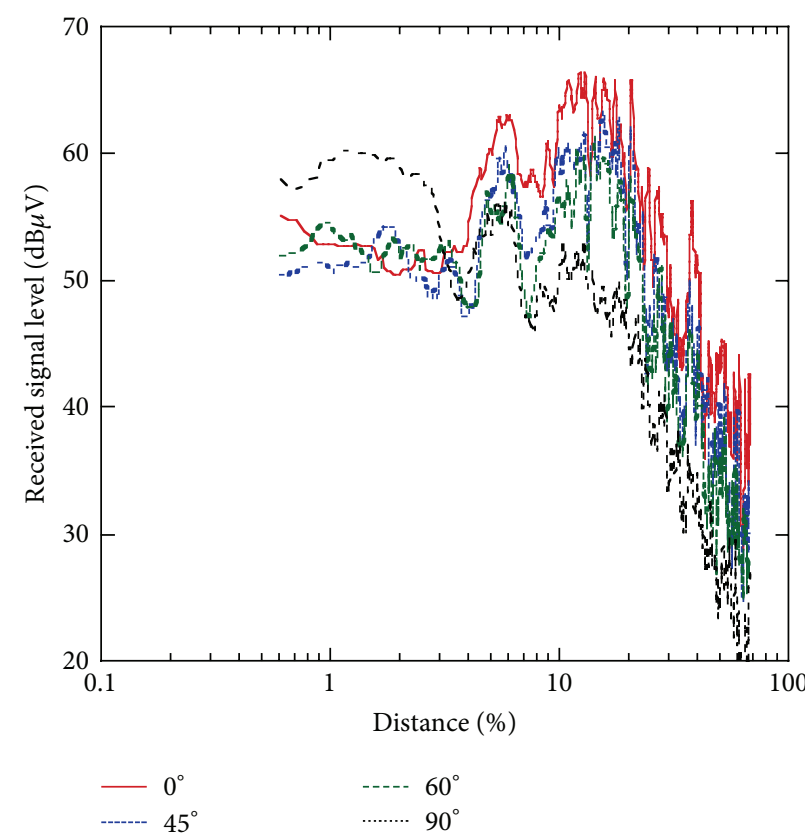

(a)

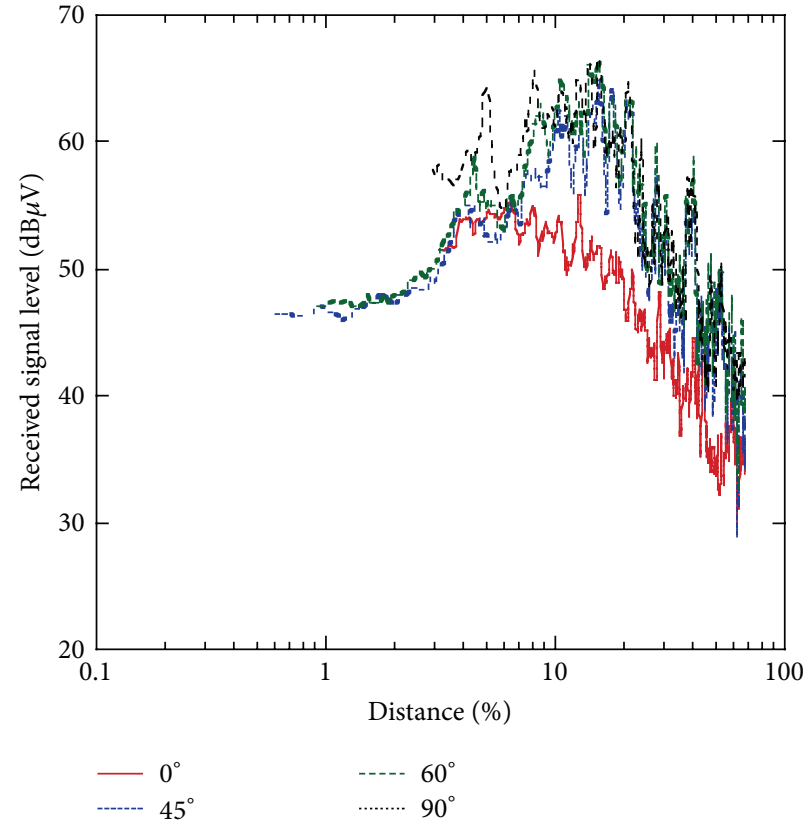

(b)

FIGURE 19: Received-signal level. (a) Collinear with tilt beam (V_tilt_antenna). (b) DSCA array with tilt beam (H_tilt_antenna).

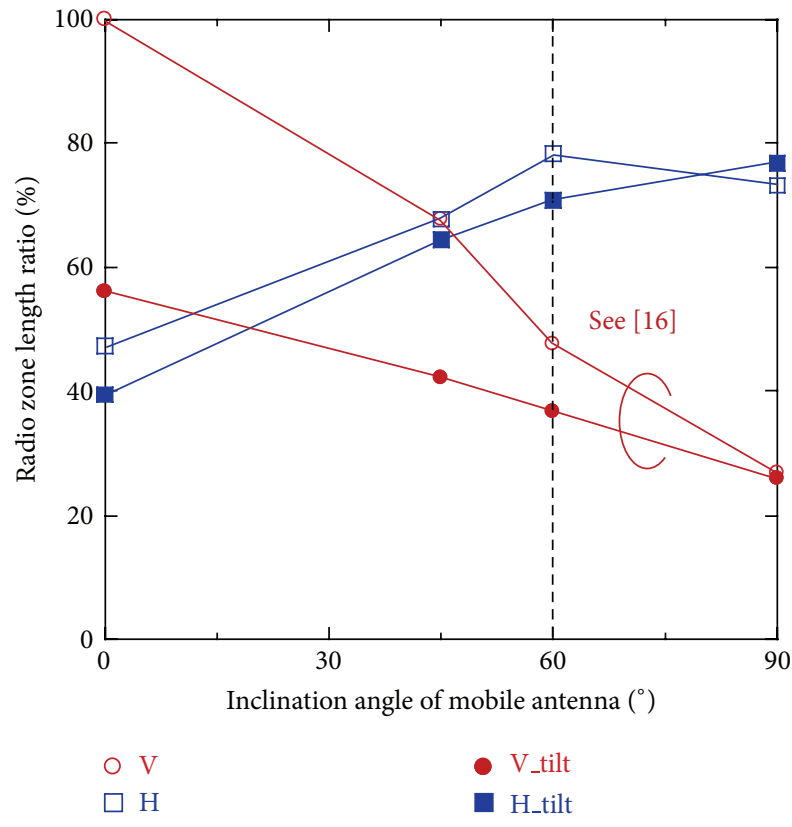

FIGURE 20: RZLR versus inclination angle of mobile antenna.

polarized omnidirectional antenna. Horizontally polarized omnidirectional mobile antennas should be used for horizontally polarized omnidirectional diversity BS antennas. The superiority in polarization of radio waves in land mobile communication systems remains as an important research issue to be addressed in future study.

\section{Conclusion}

This paper addressed the problem of CCI generated in a mixed cell architecture in PHS adopting the TDMA/TDD scheme by employing horizontally polarized omnidirectional diversity antennas that permit the PHS BS antennas established at high elevations to reduce the level of the CCI. In association with the CCI reduction technique using the horizontally polarized diversity antennas in IRBSs, the concept of polarization arrangement of the BS antennas in a mixed cell architecture was introduced. The polarizations in this type of architecture are assigned such that the IRBSs at high elevations separately use horizontally polarized omnidirectional antennas with/without beam tilt and the low elevation IBSs use vertically polarized omnidirectional antennas with/without beam tilt.

We applied a dielectric-loaded slotted-cylinder antenna (DSCA) to horizontally polarized omnidirectional array antennas in a height-diversity configuration with a high gain comparable to that of collinear antennas to reduce the CCI. The measurements of the CCI reduction effect conducted in a suburban area clarified that the DSCA array antennas with beam tilt greatly reduce the CCI level by approximately $23 \mathrm{~dB}$ due to the combined effects of both the beam tilt and polarization orthogonal to the polarization of the CCI waves. At the same time, the RZL measurements conducted on an urban street identified that the DSCA diversity antennas simultaneously achieve higher RZLs compared to those of collinear antennas. The average RZLRs with respect to the inclination angle of mobile antennas for horizontally polarized omnidirectional diversity antennas without/with 
beam tilt are 66.6 and $62.8 \%$, and greater than those of collinear antennas without/with beam tilt, 60.5 and $40.2 \%$, respectively.

It is concluded that horizontally polarized diversity antennas are superior to vertically polarized diversity antennas or collinear antennas if we take into account that the users do not use handsets in a strictly vertically hand held position but decline the units to various angles. It was demonstrated through measurement that the concepts of the CCI reduction technique for IRBSs set at high elevations using horizontally polarized diversity antennas with beam tilt and a polarization arrangement of BS antennas in a mixed cell architecture are valid and applicable to actual PHS scenarios.

\section{Disclosure}

The authors hereby declare that they have no financial relationships relevant to this paper to disclose.

\section{Conflict of Interests}

The authors also declare that they have no conflict of interests relevant to this paper.

\section{References}

[1] J. E. Padgett, C. G. Gunther, and T. Hattori, "Overview of wireless personal communications," IEEE Communications Magazine, vol. 33, no. 1, pp. 28-41, 1995.

[2] S. Yan and L. Shiming, "The development analysis of PHS in china," in Proceedings of the WRI International Conference on Communications and Mobile Computing (CMC '09), vol. 1, pp. 485-490, Yunnan, China, January 2009.

[3] O. Momtahan and H. Hashemi, "A comparative evaluation of DECT, PACS, and PHS standards for wireless local loop applications," IEEE Communications Magazine, vol. 39, no. 5, pp. 156-163, 2001.

[4] Y. Kawahara, H. Hosaka, and K. Sakata, "Positioning system using PHS and a radio beacon for logistics," in Proceedings of the IEEE International Conference on Automation and Logistics (ICAL '08), pp. 92-95, September 2008.

[5] H. Wang and Q. Chen, "Discussion of ETC online system based on PHS," in Proceedings of the International Conference on Services Systems and Services Management (ICSSSM '05), vol. 2, pp. 961-965, Chongqing, China, June 2005.

[6] S. Kozono and A. Taguchi, "Mobile propagation loss and delay spread characteristics with a low base station antenna on a urban road," IEEE Transactions on Vehicular Technology, vol. 42, no. 1, pp. 103-109, 1993.

[7] K. Nishimori, K. Cho, Y. Takatori, and T. Hori, "Two base station configuration using an adaptive array at elevated locations for microcell systems," IEICE Transactions on Communications, vol. E83-B, no. 8, pp. 1688-1696, 2000.

[8] G. V. Tsoulos, M. A. Beach, and S. C. Swales, "Application of adaptive antenna technology to third generation mixed cell radio architectures," in Proceedings of the 44th IEEE Vehicular Technology Conference, vol. 1, pp. 615-619, Stockholm, Sweden, June 1994.

[9] K. Fujimoto and J. R. James, Mobile Antenna Systems Handbook, Artech House, Boston, Mass, USA, 1994.
[10] J. Niemelä and J. Lempiäinen, "Impact of mechanical antenna downtilt on performance of WCDMA cellular network," in Proceedings of the 59th IEEE Vehicular Technology Conference (VTC '04), pp. 2091-2095, Milan, Italy, May 2004.

[11] M. Pettersen, L. E. Braten, and A. G. Spilling, "Automatic antenna tilt control for capacity enhancement in UMTS FDD," in Proceedings of the 60th IEEE Vehicular Technology Conference (VTC '04), vol. 1, pp. 280-284, Los Angeles, Calif, USA, September 2004.

[12] M. Garcia-Lozano and S. Ruiz, "Effects of downtilting on RRM parameters," in Proceedings of the 15th IEEE International Symposium on Personal, Indoor and Mobile Radio Communications (PIMRC '04), vol. 3, pp. 2166-2170, Barcelona, Spain, September 2004.

[13] I. Siomina, "P-CPICH power and antenna tilt optimization in UMTS networks," in Proceedings of the Advanced Industrial Conference on Telecommunications/Service Assurance with Partial and Intermittent Resources Conference/E-Learning on Telecommunications Workshop (AICT/SAPIR/ELETE '05), pp. 268-273, Lisbon, Portugal, July 2005.

[14] Y. Akaiwa, H. Andoh, and T. Kohama, "Autonomous decentralized inter-base-station synchronization for TDMA microcellular systems," in Proceedings of the 41st IEEE Vehicular Technology Conference, pp. 257-262, St. Louis, Mo, USA, May 1991.

[15] S. Ariyavisitakul, R. C. Lau, and H. W. Arnold, "Network synchronisation of radio ports in wireless personal communications," Electronics Letters, vol. 28, no. 25, pp. 2312-2314, 1992.

[16] A. Ando, A. Kondo, and S. Kubota, "A study of radio zone length of dual-polarized omnidirectional antennas mounted on rooftop for personal handy-phone system," IEEE Transactions on Vehicular Technology, vol. 57, no. 1, pp. 2-10, 2008.

[17] K. Cho, T. Hori, and K. Kagoshima, "Effectiveness of fourbranch height and polarization diversity configuration for street microcell," IEEE Transactions on Antennas and Propagation, vol. 46, no. 6, pp. 776-781, 1998.

[18] T. Taga and K. Tsunekawa, "Performance analysis of a built-in planar inverted $\mathrm{F}$ antenna for $800 \mathrm{MHz}$ band portable radio units," IEEE Journal on Selected Areas in Communications, vol. 5, no. 5, pp. 921-929, 1987.

[19] A. Ando, Y. Honma, and K. Kagoshima, "An electromagnetically coupled microstrip antenna with a rotatable patch," in Proceedings of the IEEE International Symposium on Antennas and Propagation (AP-S '95), vol. 1, pp. 354-357, Port Beach, Calif, USA, June 1995.

[20] T. Taga and K. Tsunekawa, "A built-in antenna for $800 \mathrm{MHz}$ band portable radio units," in Proceedings of the International Symposium on Antennas and Propagation (ISAP '85), pp. 425428, Kyoto, Japan, August 1985.

[21] H. L. Bertoni, Radio Propagation for Modern Wireless Systems, Prentice Hall, Upper Saddle River, NJ, USA, 2000. 

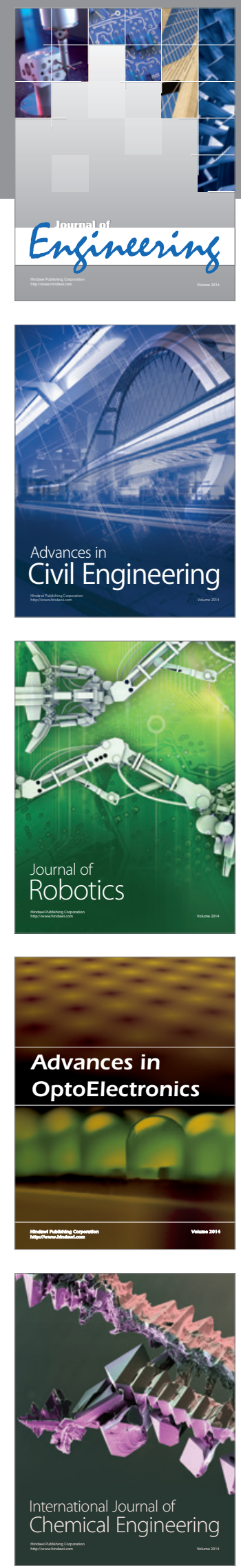

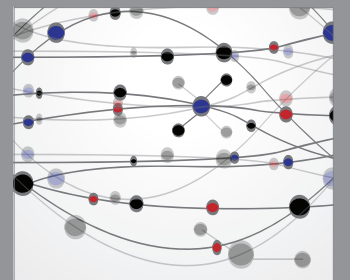

The Scientific World Journal
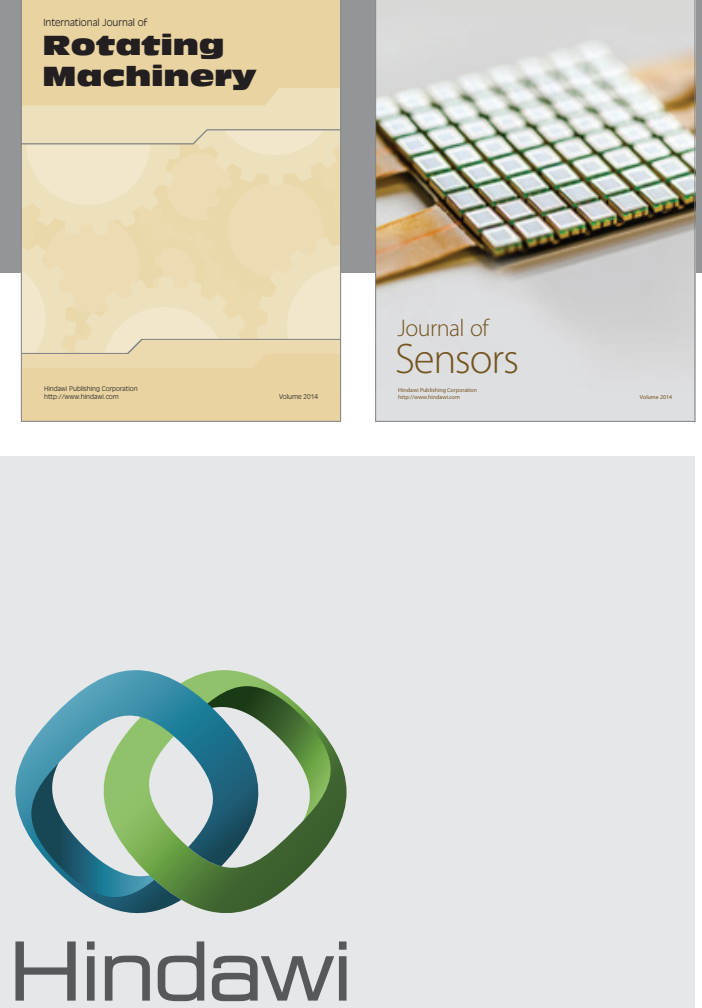

Submit your manuscripts at http://www.hindawi.com
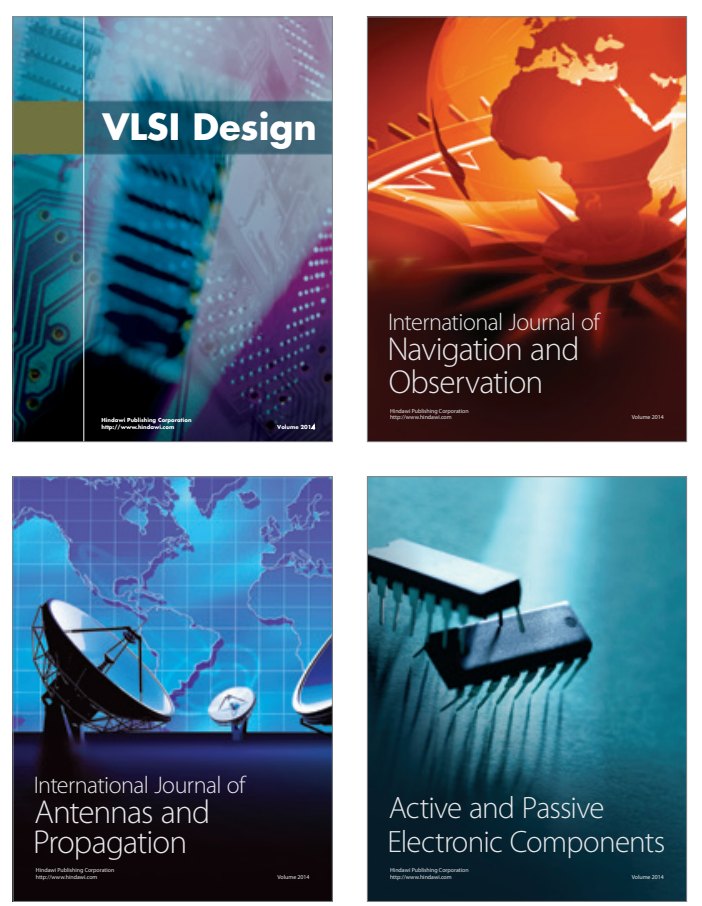
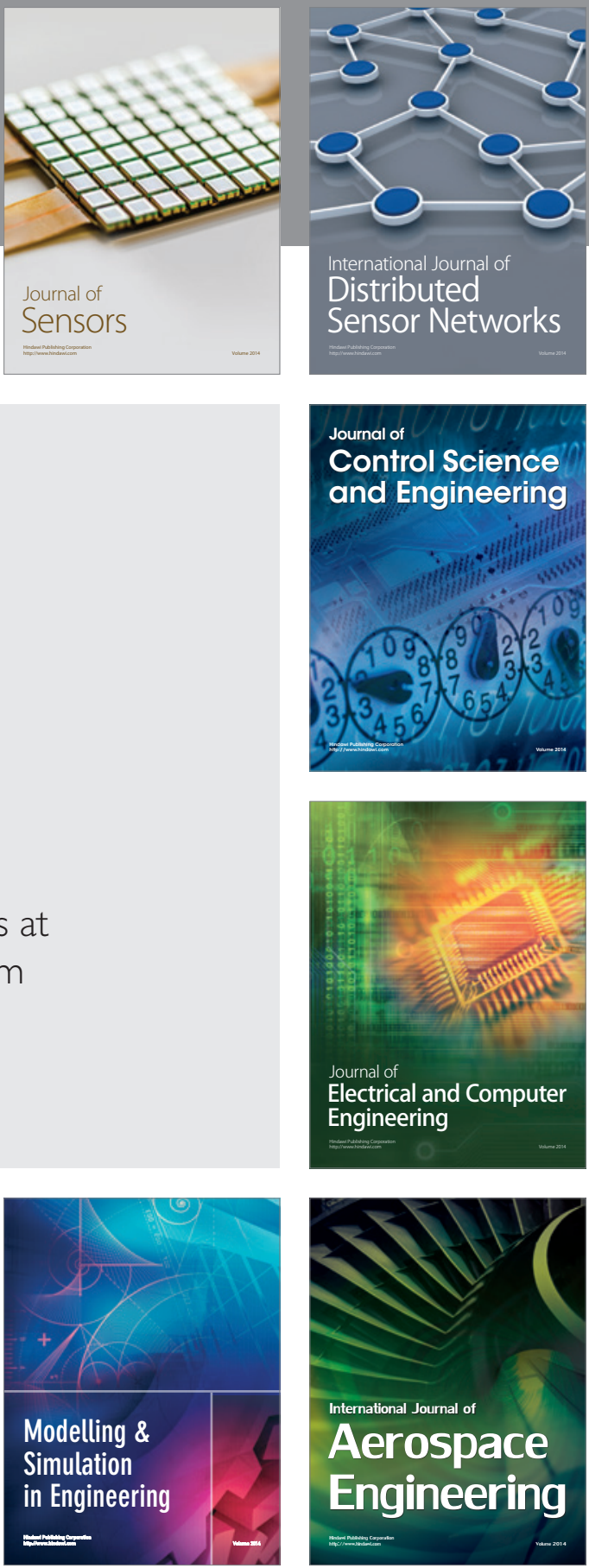

Journal of

Control Science

and Engineering
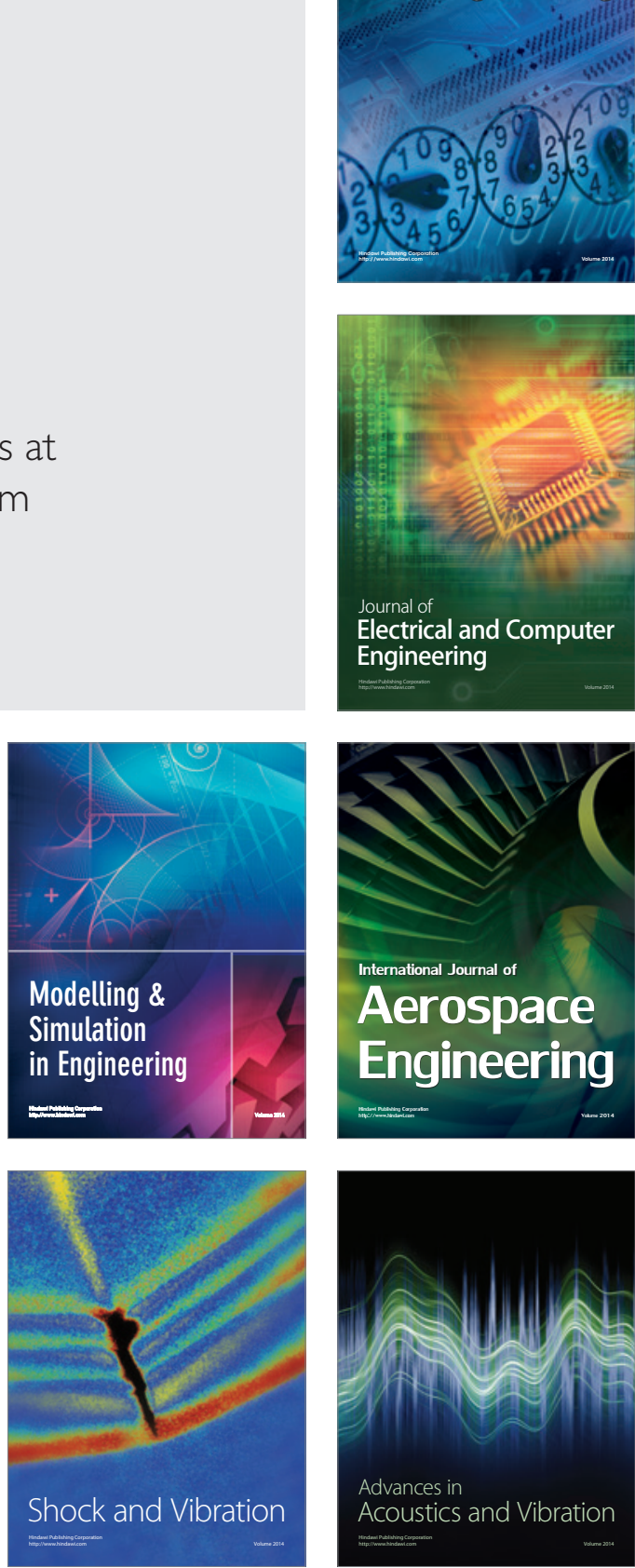